\title{
Efectos de la microestructura en las propiedades magnéticas de imanes híbridos de $\mathrm{SrFe}_{12} \mathrm{O}_{19} / \mathrm{MQP}-\mathrm{Q}$
}

\author{
D. BUENO BAQUÉS, J. A. MATUTES AQUINO, V. CORRAL FLORES Y O. AYALA VALENZUELA \\ Centro de investigación en Materiales Avanzados, S.C. \\ Miguel de Cervantes \#120, Complejo Industrial Chihuahua. \\ Chihuahua, Chih. 31109. México
}

\begin{abstract}
En este trabajo se estudiaron la microestructura y propiedades magnéticas de imanes híbridos moldeados por compresión, constituidos por mezclas de Ferrita de Estroncio $\left(\mathrm{SrFe}_{12} \mathrm{O}_{19}\right)$ y $\mathrm{MQP}-\mathrm{Q}\left(\mathrm{Nd}_{2} \mathrm{Fe}_{14} \mathrm{~B}+\alpha \mathrm{Fe}\right)$ embebidas en una matriz de Polietileno Lineal de Baja Densidad (LLDPE). Se comparan las propiedades de las muestras con una relación \% en peso de ferrita / \% en peso de MQP-Q de 4/82, 48/6 y 26/68 con polvos de los materiales de partida. La microestructura se estudió por microscopía electrónica de barrido y microanálisis elemental. Las interacciones magnéticas fueron estudiadas por medio de curvas de desmagnetización isotérmica (IRM) y de desmagnetización DC (DCD). Se observan interacciones inicialmente positivas, que decrecen pasando por cero, con un mínimo negativo, a medida que se incrementa el campo magnético. Se discute el efecto de la microestructura en las curvas de $\Delta \mathrm{M}$.
\end{abstract}

Palabras clave: Materiales magnéticos, Imanes hibridos, Interacciones magnéticas.

The effect of the microstructure in the magnetic properties of $\mathrm{SrFe}_{12} \mathrm{O}_{19} / \mathrm{MQP}-\mathrm{Q}$ hybrid bonded magnets.

Magnetic properties in Hybrid magnets, compression-molded consisting on mixtures of $\mathrm{SrFe}_{12} \mathrm{O}_{19}$ ferrite and MQP-Q $\left(\mathrm{Nd}_{2} \mathrm{Fe}_{14} \mathrm{~B}+\alpha \mathrm{Fe}\right)$ embedded in LLDPE were studied. Samples with the ratio $\%$ wt ferrite $/ \%$ wt MQP-Q $4 / 82,48 / 6$ and $26 / 68$ are compared to powders of pure starting magnetic materials. Microstructure was investigated by SEM and EDS. Magnetic interactions have been studied by isothermal remanence (IRM), dc-demagnetization remanence (DCD) curves. Hybrid samples show interactions initially positive, falling rapidly through zero, with a negative minimum, as the magnetic field increases. The effect of the microstructure in the $\Delta \mathrm{M}$ curves is discussed.

Keywords: Magnetic materials, Hybrid magnets, Magnetic Interactions.

\section{INTRODUCCIÓN}

Los imanes compuestos constituyen uno de los renglones de mayor crecimiento dentro del mercado de los imanes permanentes. Estos se producen encapsulando un polvo magnético o carga magnética en una matriz polimérica. $\mathrm{Su}$ alta importancia tecnológica radica en la posibilidad de obtener piezas de forma muy variada usando prácticamente los mismos métodos utilizados para el procesamiento de plásticos.

En particular dentro de este campo resalta la posibilidad de obtener imanes híbridos compuestos por distintas cargas magnéticas, a partir de los cuales es posible obtener imanes con propiedades magnéticas intermedias. Recientemente se han estudiado sistemas de este tipo formados por el compuesto intermetálico $\mathrm{Nd}_{2} \mathrm{Fe}_{14} \mathrm{~B}$ y por ferrita de Bario $\mathrm{BaFe}_{12} \mathrm{O}_{19}$ con propiedades magnéticas interesantes [1].

La posibilidad de controlar la carga magnética, y con ello la distancia media entre las partículas magnéticas en los imanes compuestos híbridos ofrece buen sistema para el estudio de las interacciones magnéticas entre las partículas. Una descripción de estas interacciones puede ser obtenida en términos de las curvas de $\Delta \mathrm{m}$, obtenidas a partir de las remanencias en los procesos de desmagnetización en corriente directa (DCD) y magnetización isotérmica remanente (IRM) [2].

El presente trabajo aborda el estudio de los efectos microestructurales en las interacciones magnéticas en imanes híbridos obtenidos a partir de polvos de ferrita de estroncio $\left(\mathrm{SrFe}_{12} \mathrm{O}_{19}\right)$ y polvos de $\mathrm{Nd}_{2} \mathrm{Fe}_{14} \mathrm{~B}+\alpha \mathrm{Fe}$.

\section{MATERIALES Y MÉTODOS}

Las muestras de Imanes Híbridos consideradas en el presente trabajo fueron desarrolladas en el Centro de Investigación en Química Aplicada (CIQA). A partir de polvo de ferrita de estroncio $\mathrm{SrFe}_{12} \mathrm{O}_{19}\left(\mathrm{M}_{\mathrm{r}} / \mathrm{M}_{\mathrm{s}}=0.5, \mathrm{Hc}=2.1 \mathrm{kOe}\right)$ fabricado por Hoosier Magnetics Inc., de polvo de MQP-Q $\left(\mathrm{Nd}_{2} \mathrm{Fe}_{14} \mathrm{~B}+\alpha \mathrm{Fe} \mathrm{M}_{\mathrm{r}} / \mathrm{M}_{\mathrm{s}}=0.61, \mathrm{Hc}=3.5 \mathrm{kOe}\right)$ fabricado por Magnequench International Inc. y de Polietileno Lineal de Baja Densidad (LLDPE) modificado con Anhídridro Maleico Bynel fabricado por Dunpont se obtuvieron imanes isotrópicos mediante la técnica de moldeo por compresión [3]. Para comparar el efecto de la concentración de los componentes sobre las propiedades de los imanes se seleccionaron muestras con las siguientes relaciones en peso: muestra M1, $4 \%$ Ferrita $\mathrm{Sr}+82 \% \mathrm{MQP}-\mathrm{Q}+14 \%$ LLDPE (densidad $\left.\rho_{\mathrm{M} 1}=3.40 \mathrm{~g} \mathrm{~cm}^{-3}\right)$, muestra M2, $48 \%$ Ferrita de $\mathrm{Sr}+6 \% \mathrm{MQP}-\mathrm{M}+46 \%$ LLDPE (densidad $\rho_{\mathrm{M} 2}=1.56 \mathrm{~g} \mathrm{~cm}^{-3}$ ) y muestra M3, $26 \%$ Ferrita Sr+ 68 $\%$ MQP-Q $+6 \%$ LLDPE (densidad $\rho_{\mathrm{M} 3}=4.10 \mathrm{~g} \mathrm{~cm}^{-3}$ ). 
La microestructura se estudió usando un microscopio electrónico de barrido Jeol equipado con espectroscopia por discriminación de energía de rayos X (EDS) EDAX.

Las propiedades magnéticas se determinaron en un magnetómetro de muestra vibrante LDJ 9600 con un campo máximo de $25 \mathrm{kOe}$. Todas las muestras fueron cortadas en forma de agujas $(0.5 \sim 0.6 \times 6 \mathrm{~mm})$ con una relación diámetro: longitud igual a 1:10 y medidas con la longitud paralela al campo magnético aplicado. En todos los casos, el campo interno $\mathrm{H}_{\mathrm{i}}$ fue calculado usando el factor de desmagnetización apropiado para la geometría de las muestras.

\section{RESULTADOS Y DISCUSIÓN}

La tabla 1 muestra los valores para la magnetización de saturación $M_{s^{\prime}}$ la magnetización remanente $M_{r}$ y la relación $\mathrm{M}_{\mathrm{r}} / \mathrm{M}_{\mathrm{s}}$ obtenidos a partir de los lazos de histéresis de las muestras estudiadas (Figura 1). La magnetización

TABLA I. VALORES PARA LA MAGNETIZACIÓN DE SATURACIÓN $\mathrm{M}_{\mathrm{S}^{\prime}}$ LA MAGNETIZACIÓN REMANENTE $\mathrm{M}_{\mathrm{p}}$ Y LA RELACIÓN $\mathrm{M}_{\mathrm{R}} / \mathrm{M}$

\begin{tabular}{|c|c|c|c|c|}
\hline Muestra & $4 \pi \mathrm{M}_{\mathrm{s}}(\mathrm{kG})$ & $4 \pi \mathrm{M}_{\mathrm{r}}(\mathrm{kG})$ & $\mathrm{M}_{\mathrm{r}} / \mathrm{M}_{\mathrm{s}}$ & $\mathrm{iHc}(\mathrm{Oe})$ \\
\hline $\mathrm{M} 1$ & 5.41 & 3.5 & 0.65 & 3718 \\
\hline $\mathrm{M} 2$ & 1.25 & 0.51 & 0.41 & 4097 \\
\hline $\mathrm{M} 3$ & 6.69 & 3.81 & 0.57 & 3658 \\
\hline
\end{tabular}

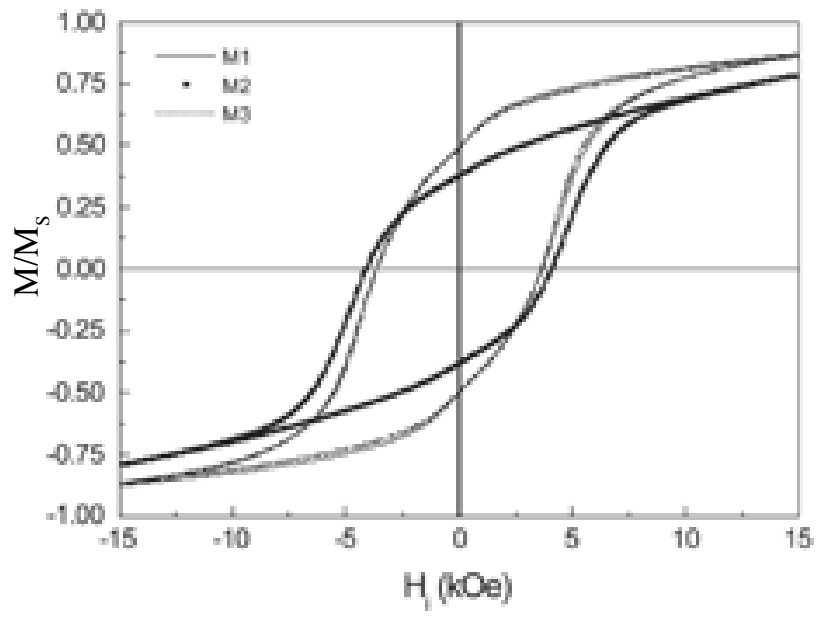

Fig. 1.- Lazos de Histéresis para las muestras M1, M2 y M3. específica de saturación aumenta con el contenido de la carga magnética, mientras que los valores de coercitividad permanecen prácticamente constantes. En la muestra M2 la baja concentración de carga magnética provoca una baja magnetización de saturación, mientras el mejor aislamiento de las partículas por parte del polímero provoca un incremento en el campo coercitivo. En las muestras M1 y M3, con mayor proporción de MQP-Q, se observa un aumento en el coeficiente $\mathrm{M}_{\mathrm{r}} / \mathrm{M}_{\mathrm{s}}$ por encima del valor de 0.5 esperado para imanes isotrópicos con partículas que no interactúan entre si. Este aumento refleja el acoplamiento de intercambio entre los nanogranos de $\mathrm{Nd}_{2} \mathrm{Fe}_{14} \mathrm{~B}$ y $\alpha \mathrm{Fe}$ en los granos de MQP-Q.

La Figura 2 muestra las micrografías electrónicas de barrido para las muestras. En estas es posible observar cómo las partículas de MQP-Q y ferrita de Sr se distribuyen aleatoriamente en la matriz formada por el polímero. Las micrografías reflejan el elevado desorden estructural introducido durante el proceso de obtención. La micrografía de la muestra M2 refleja la baja concentración de carga magnética y el asilamiento de las partículas. Las muestras M1 y M3, salvo diferencias en la concentración de los componentes, muestran una microestructura muy similar.

Para estudiar las interacciones magnéticas se midieron las curvas de remanencia en desmagnetización DC (siglas DCD en inglés) y las curvas de magnetización remanente isotérmica (siglas IRM en inglés).

Para todo sistema de partículas monodominio que no interactúan se cumple la relación de Wohlfarth que establece que $M_{d}(H)=M_{r}(\infty)-2 M_{r}(H)$ [4]. $M_{d}(H)$ corresponde a la magnetización remanente obtenida a partir de las curvas DCD partiendo del estado de saturación en campo positivo y medida como función del incremento negativo del campo hasta el estado de saturación negativa. $\mathrm{M}_{\mathrm{r}}(\infty)$ corresponde al valor de remanencia del lazo mayor de histéresis. $M_{r}(H)$ corresponde al valor de la magnetización remanente partiendo del estado desmagnetizado AC como función del incremento del campo aplicado hasta el estado de saturación positiva.

$\Delta \mathrm{m}(\mathrm{H})=\mathrm{M}_{\mathrm{d}}(\mathrm{H})-\left[\mathrm{M}_{\mathrm{r}}(\infty)-2 \mathrm{M}_{\mathrm{r}}(\mathrm{H})\right]$ mide la desviación de la relación de Wohlfarth. Para un sistema de partículas que interactúan entre sí es posible estimar el tipo y magnitud de las interacciones presentes a partir del valor de $\Delta \mathrm{m}(\mathrm{H})$. Valores positivos de $\Delta \mathrm{m}(\mathrm{H})$ corresponden a interacciones magnetizantes mientras que valores negativos se asocian a interacciones desmagnetizantes.

La Figura 3 muestra las curvas de $\Delta \mathrm{m}(\mathrm{H})$ para las muestras M1, M2 y M3. Se observan interacciones inicialmente positivas (magnetizantes) que disminuyen rápidamente tomando

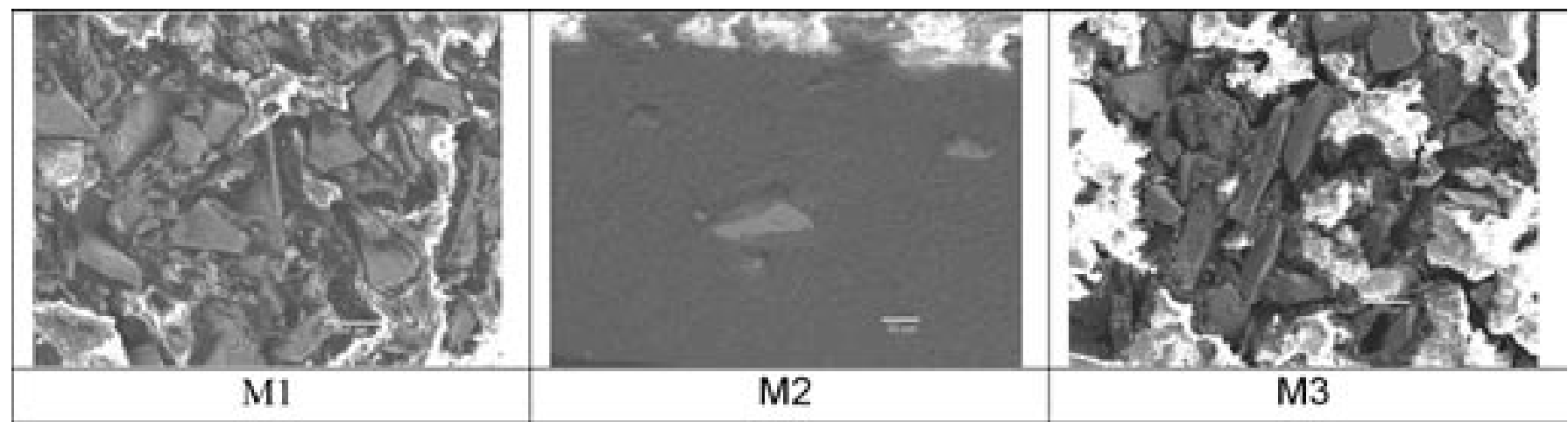

Fig. 2.- Micrografías de barrido de las muestra M1, M2 y M3 


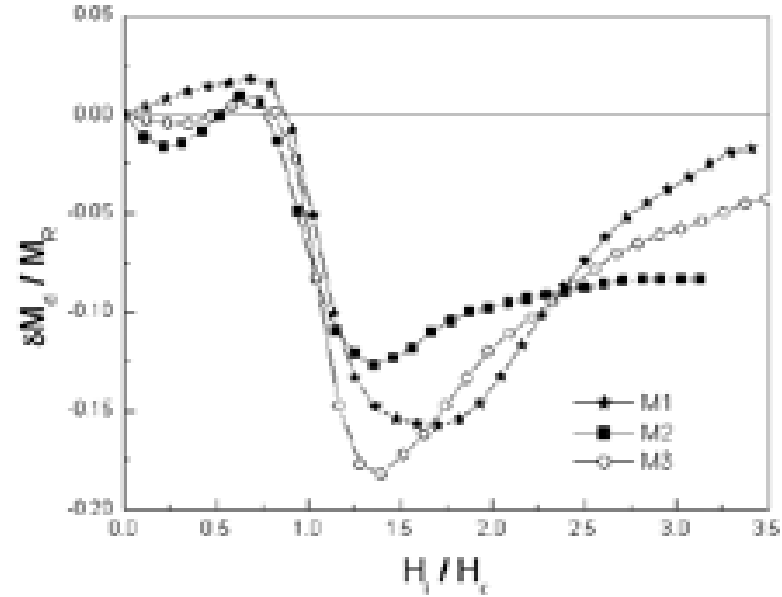

Fig. 3.- Curvas de $\Delta \mathrm{M}(\mathrm{H})$ para las muestras $\mathrm{M} 1$, $\mathrm{M} 2$ y $\mathrm{M} 3$

valores negativos (desmagnetizantes) a medida que el campo magnético aumenta. Dicho comportamiento puede asociarse al elevado desorden estructural presente en las muestras y la existencia de regiones donde se presentan interacciones que conducen a una inversión cooperativa de la magnetización [5,6]. En la muestra M5 con un contenido más alto de MQP$\mathrm{Q}$, los valores más elevados de $\Delta \mathrm{m}(\mathrm{H})$ en la región con interacciones magnetizantes reflejan el acoplamiento por intercambio entre los nanogranos de $\mathrm{NdFeB}$ y $\alpha \mathrm{Fe}$.

En estos compuestos híbridos isotrópicos es posible suponer que la componente reversible de la magnetización $\mid$ Mrev | resulta del movimiento reversible inicial de las paredes de dominio en la ferrita de Sr, limitado por centros de anclaje y posteriormente, para campos mayores, de la rotación reversible de la magnetización en cada grano de ferrita y en los nanogranos de MQP-Q ( $\alpha$ Fe y NdFeB). Cuando se aplica un campo al estado inicial o de remanencia, el sistema adquiere una cierta configuración en la orientación de los momentos magnéticos de cada grano. Esta configuración determina los valores de $\mathrm{Mr}$ y $\mathrm{Md}$. Cuando el campo se reduce a cero, los momentos magnéticos en los granos tienden a rotar hacia sus direcciones de fácil magnetización. Sin embargo, los centros de anclaje en los granos de ferrita de $\mathrm{Sr}$ y el acoplamiento de intercambio entre los nanogranos de $\alpha \mathrm{Fe}$ y NdFeB impiden la rotación completa del volumen de cada grano. En virtud de esto, el grado de acoplamiento entre los granos, la magnitud del campo aplicado, la concentración de cada componente, junto al desorden estructural determinan el comportamiento de las curvas de $\Delta \mathrm{m}(\mathrm{H})$.

\section{CONCLUSIONES}

Las interacciones magnéticas en los imanes híbridos formados por MQP-Q + ferrita de estroncio en una matriz de polietileno lineal de baja densidad resultan dependientes de la composición y microestructura introducida por el proceso de obtención. La forma de las curvas de $\Delta \mathrm{m}$ muestra un predominio de interacciones de tipo desmagnetizantes, reflejando el elevado desorden estructural presente en las muestras y la intensidad de las interacciones magnetostáticas entre los granos. Para las muestras con mayor contenido de MQP-Q se obtienen interacciones magnetizantes muy débiles para campos magnéticos menores que el coercitivo, reflejando el acoplamiento de intercambio entre los nanogranos de $\mathrm{NdFeB}$ y $\alpha \mathrm{Fe}$.

\section{AGRADECIMIENTOS}

Los autores agradecen el apoyo del CONACYT por medio del proyecto de investigación 32621-U “Magnetometría de Altos Campos Magnéticos".

\section{BIBLIOGRAFÍA}

1. M. Emura, A.C. Neiva, F.P. Missell, K.L. Babcock, J. Ormerod, S Constantinides, "Magnetization processes in hybrid magnets", J. Appl. Phys., 83 [11] 7127-7129 (1998).

2. G. Bertotti, Part V "Preisach Systems" pp. 433-534 in Hysteresis in Magnetism, Academic Press series in Electromagnetism, Edited by Isaak Mayergoyz. Academic Press, San Diego, CA. USA, 1998

3. J. Matutes Aquino, D. Ríos Jara, O. Ayala Valenzuela, P. Sifuentes Gallardo, L.F. Ramos de Valle, O.S. Rodríguez Fernández., “Composition dependence of the magnetic properties of bonded magnets of strontium hexaferritepolyvinyl chloride", Polymer Composites, 215 734-738 (2000)

4. E. P. Wohlfarth., "Relations between different modes of acquisition of the remanent magnetization of ferromagnetic particles", J. Appl. Phys. 293 595-596 (1958)

5. M. P. Morales, K. O'Grady, P.I. Mayo, “Textura and interactions effects in Barium ferrite media". IEEE Trans. Magn. 316 2904-2906 (1995)

6. V. Patel, M. El-Hilo, K. O'Grady, R. W. Chantrell, "Fluctuation field of reversal in oriented and non-oriented barium hexaferrite media", IEEE Trans. Magn. 296 3622- 3624 (1993).

Recibido: 15.04 .04

Aceptado: 14.03 .05 